\title{
Using Bayesian Networks to Model Emergency Medical Services
}

\author{
Silvia Acid ${ }^{1}$, Luis M. de Campos $^{1}$, Susana Rodríguez ${ }^{2}$, José María Rodríguez ${ }^{2}$, \\ and José Luis Salcedo ${ }^{2}$ \\ 1 Dpto de Ciencias de la Computación e I.A., Universidad de Granada \\ 18071 - Granada, Spain \\ acid@decsai.ugr.es, lci@decsai.ugr.es \\ 2 Hospital Universitario Virgen de las Nieves \\ 18014 - Granada, Spain \\ \{susana, joser\}@hvn.sas.cica.es
}

\begin{abstract}
Due to the uncertain nature of many of the factors that influence on the performance of an emergency medical service, we propose using Bayesian networks to model this kind of systems. We use an algorithm for learning Bayesian networks to build the model, from the point of view of a hospital manager, and apply it to the specific case of a spanish hospital. We also report the results of some preliminary experimentation with the model.
\end{abstract}

Keywords: Bayesian networks, Learning algorithms, Emergency medical services, Management problems.

\section{Introduction}

Health-care systems are complex and depend on organizational, economical and structural factors. The availability of appropriate tools for their representation would allow to study and understand the interactions among the different elements that determine their behaviour, as well as to analyze some alternatives to improve their performance. As many of the factors that influence on the performance of a health-care system are of a uncertain nature, Bayesian networks [Pea88] could play an important role in their study. In this paper we introduce a representation model, based on Bayesian networks, applied to the specific case of an emergency medical service. This model has been obtained, from real data recorded at the hospital "Virgen de las Nieves", using an algorithm for learning Bayesian networks.

The paper is structured as follows: In Section 2 we describe the problem we are going to study and the available data. In Section 3 we briefly comment on the learning algorithm that we have used to build the model. Section 4 describes the network obtained and the results of several experiments, which try to assess the quality of the model from a classification-oriented perspective. In Section 6 we offer some conclusions and proposals for future work. 
Table 1. Variables

\begin{tabular}{|c|c||c|c|}
\hline Variable & n. of values & Variable & n. of values \\
\hline Financing & 11 & Identification & 6 \\
Day & 7 & Duration of the Stay & 3 \\
Shift & 3 & Cause of Discharge & 11 \\
Cause of Admission & 8 & Medical Service & 36 \\
Pathology & 7 & Centre & 3 \\
P10 & 2 & & \\
\hline
\end{tabular}

\section{The Problem and the Data Set}

As we have already commented, we want to model some aspects of the healthcare system for patients that arrive to the emergency department of a hospital. Our first objective is to better understand the interactions between some of the factors that shape this system, and obtain a model that describes reasonably these interactions. Afterwards, the model could be used to estimate the different probability distributions of some variables of interest in different contexts, or even to make predictions about these variables given some partial evidence. Our point of view is not clinical but management oriented, i.e., we try to assist to the hospital manager in organizational and economical questions (e.g., possible redistribution or reinforcement of personnel and infrastructure).

From the set of variables which are collected when a patient enters in the emergency department, the eleven variables displayed in Table 1 were selected. The number of possible values for each variable is also shown in the table. We used a database containing 31937 records, corresponding to all the arrivals to the emergency departments of the hospital "Virgen de las Nieves" at Granada, from $01 / 01 / 2001$ to $20 / 02 / 2001$.

Financing represents the type of entity that supports the expenses (Social Security, Insurance companies, International agreements, ...). Day is the day of the week in which the patient arrives to the emergency department. Shift corresponds to the arrival time of the patient, discretized into 3 values, representing the three different horary periods of the day: morning (8:01-15:00), evening (15:01-22:00) and night (22:01-8:00). Cause of Admission codifies 8 different values ${ }^{1}$. Pathology includes Common Disease, Common Accident, Industrial Accident, Traffic Accident, Aggression, Self-inflicted Lesion and Other. P10 represents whether the patient was sent to the emergency medical service by a family doctor. Identification codifies the type of identification document of the patient (Identity Card, Social Security Card, Passport, Oral Identification, Other and Unidentified). Duration of the Stay is the lenght of time (in hours) that the patient stayed in the emergency department, discretized into 3 values, considered meaningful by the physicians (from 0 to 8 hours, from 8 to 72 hours, and more than 72 hours, which correspond to normal, complicated and anomalous

\footnotetext{
${ }^{1}$ Considered as confidential by the hospital staff.
} 
cases, respectively). Cause of Discharge represents several reasons (Return to duty, Death, Hospitalization, Transfer to another hospital, ...). Medical Service includes all the different emergency units at the hospital. Finally, Centre represents the three different emergency departments corresponding to the three centres that compose the hospital (Maternity hospital, Orthopedic Surgery and General hospital).

\section{The Algorithm for Learning Bayesian Networks}

There are many algorithms for learning the structure of a Bayesian network from a database, although they may be categorized in only two methods, those based on independence criteria [SGS93] or on scoring metrics [CH92,HGC95].

The learning algorithm that we have applied to our problem is a version of the BENEDICT (BE) algorithm ${ }^{2}[\mathrm{AC}]$. This algorithm, which searches in the space of equivalence classes of directed acyclic graphs $(\text { dags })^{3}$, is based on a hybrid methodology that shares with the methods based on scoring metrics the use of heuristic search methods to build a model and then evaluate it using a scoring metric. At the same time, the method has some similarities with those based on independence tests: it explicitly uses the conditional independencies embodied in the topology of the network to elaborate the scoring metric, and carries out conditional independence tests to limit the search effort.

The basic idea of this algorithm is to measure the discrepancies between the conditional independences represented in any given candidate network $G$ (d-separation statements) and those displayed by the database (probabilistic conditional independences). The lesser these discrepancies are, the better the network fits the data. The aggregation of all these local discrepancies results in a measure of global discrepancy between the network and the database. The local discrepancies are measured using the Kullback-Leibler cross entropy, $\operatorname{Dep}(x, y \mid Z)$, which measures the degree of dependence between two variables $x$ and $y$, given that we know the values of the subset of variables $Z$. To evaluate a network $G$, only the values $\operatorname{Dep}(x, y \mid Z)$ for pairs of non-adjacent variables in $G$, given a d-separating set for $x$ and $y$ of minimum size, $Z$ [AC96], are calculated. The main search process is greedy and only addition of arcs is permitted, although a final refining process (reinsertion of discarded arcs and pruning of inserted arcs) mitigates the irrevocable character of the whole search method.

To compute the conditional (or marginal) probability distributions stored at each node in the network, thus obtaining a complete Bayesian network, we used a maximum likelihood estimator (frequency counts).

\footnotetext{
${ }^{2}$ Acronym of BElief NEtwork DIscovery using Cut-set Techniques.

${ }^{3}$ Other versions of BENEDICT, that search in the space of dags with a given ordering of the variables, and use a slighty different metric, can be found in [AC01].
} 


\section{Results}

After running the learning algorithm we obtained the network displayed in Figure 1. We want to remark that we do not assume a causal interpretation of the arcs in the network (although in some cases this could be reasonable). Instead, we interpret the arcs as direct dependence relationships between the linked variables, and the absence of arcs means the existence of (conditional) independence relationships.

Due to space limitations, we will not comment on all the 16 arcs included in the network, but only about some of them. The dependence between Pathology and Financing is explained because the expenses are charged to different entities depending on the type of pathology (traffic accident, industrial accident,...).

Financing also depends on Identification (obviously the expenses will be charged to some entity or company only if the patient can be identified as a member of this entity). The dependence between Pathology and Cause of Admission is quite evident. The relation between Cause of Admission and Shift may be due to the fact that the reason to go to the emergency department is not homogeneous across the different hours (Shifts). The arc going from Medical Service to Centre is justified because Centre is a variable functionally dependent on Medical Service (each Centre has its own emergency medical units). The Duration of the stay at the emergency department essentially depends only on the medical unit that tended the patient and the Cause of Discharge (the seriousness of the diseases, which is strongly related with the duration of the stay, probably varies from one unit to another). In turn, these two variables are also correlated: For example, a decease as being the cause of discharge is much more unlikely for some medical units than for others.

The learned network can be used with predictive purposes, by using the inference methods (propagation of evidence) available for Bayesian networks. More precisely, from the perspective of a classification problem, we want to use the network to predict the values of some variable of interest given some evidence,

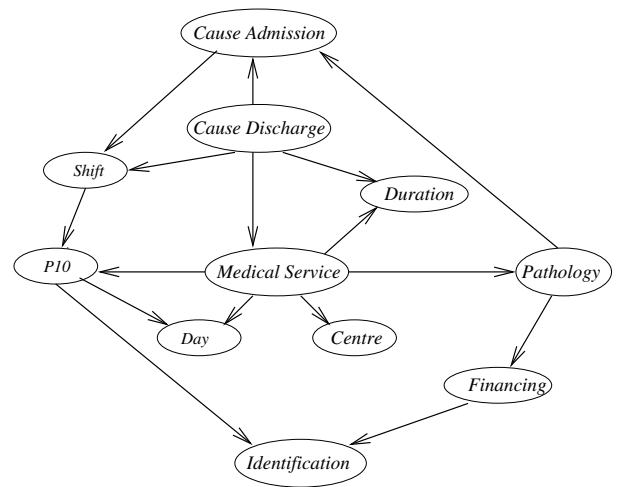

Fig. 1. Structure obtained by the BEnEDICT algorithm 
Table 2. Success percentages of classification

\begin{tabular}{|c|c|c|c|c||c|c|c|c|}
\cline { 2 - 9 } \multicolumn{1}{c|}{} & \multicolumn{3}{c||}{ Training Set } & \multicolumn{4}{c|}{ Test Set } \\
\cline { 2 - 9 } \multicolumn{1}{c|}{} & BE & $\emptyset_{\mathrm{em}}$ & NB & C4.5 & BE & $\emptyset_{\mathrm{em}}$ & NB & C4.5 \\
\hline D \% & 89.9 & 86.1 & 86.5 & 90.1 & 91.6 & 96.1 & 86.3 & 92.0 \\
MS \% & 75.9 & 30.4 & 76.0 & 75.9 & 75.0 & 31.9 & 76.0 & 76.1 \\
P \% & 85.5 & 79.6 & 85.5 & 85.5 & 85.6 & 80.7 & 85.6 & 85.6 \\
\hline
\end{tabular}

and compare the predictions obtained with the true values of this variable, thus obtaining the corresponding percentages of success. We have considered three different situations:

- Predicting the values of Duration (D \%), given evidence about the values of all the other variables, except Cause of Discharge. In this way, we try to determine the most probable duration of the stay at the emergency department before the patient is effectively discharged. This information could be useful to redistribute some resources.

- Predicting the values of Medical Service (MS \%), given evidence relative to all the remaining variables, except Pathology, Cause of Discharge and Duration, which would be unknown at the arrival time of the patient. If accurate, this prediction could serve to direct the arriving patient to the appropriate emergency unit.

- Predicting the values of Pathology (P \%) given Medical Service and Cause of Admission.

These experiments may also give us an idea of the robustness of the Bayesian network as a general classifier, as opposed to have to manage a different model for each different problem. We have computed the success percentages obtained for the network learned by BENEDICT and the empty network $\left(\emptyset_{\mathrm{em}}\right)$, which is obviously a poor model (no interaction among the variables), as well as those obtained by the Naive Bayes (NB) classifier [DH73] and C4.5 [Qui93] (a classifier based on decision trees). Table 2 displays the percentages of success obtained for classifying the same data used to build the models (training set) and also for a separate test set $^{4}$.

In general, the results obtained by $\mathrm{BE}, \mathrm{NB}$ and $\mathrm{C} 4.5$ are quite similar. With respect to predicting the Duration of the stay, note that the percentage of improvement obtained with respect to the prediction of the empty network is rather small for the training set. The reason is that the distribution of the duration of the stay is quite biased towards its first value (from 0 to 8 hours) and therefore the default rule that assigns to all the cases the 'a priori' most probable class gets a high percentage of correct classifications (in fact, in the test set, the proportion of cases with a duration of the stay from 0 to 8 hours is even greater). Similarly, we also get a moderate improvement in the prediction of the

${ }^{4}$ containing 12292 arrivals of patients, from $21 / 02 / 01$ to $10 / 03 / 01$. 
Table 3. Posterior distribution of Shift given P10 and Day

\begin{tabular}{|l|c|c|c|}
\hline Configuration & Morning & Evening & Night \\
\hline P10=no, $\forall$ Day & 0.34 & 0.47 & 0.18 \\
P10=yes, Day=Weekday & 0.47 & 0.36 & 0.17 \\
P10=yes, Day=Weekend & 0.44 & 0.37 & 0.18 \\
\hline
\end{tabular}

Pathology, when using any of the models instead of the 'a priori' distribution. In this case the distribution of pathology is also quite biased towards its first value (common disease). The problem of predicting the Medical Service involved is more difficult, and in this case BE, NB and C4.5 considerably outperform the prediction of the empty network.

The network model can also be used to compute the posterior probability of any variable in different contexts. For example, we have calculated the posterior probability distribution of Shift given P10 and Day for all the possibles values of these two variables. Table 3 summarizes the results.

It is interesting to note how the arrival pattern to the emergency medical services is quite homogeneous across the different days (including weekend), but this pattern is different depending on patients having a P10 document or not. As expected, patients having a P10 document arrive more frequently in the morning.

\section{Concluding Remarks}

The complexity of the health-care systems requires appropriate tools for their representation, study and optimization. Bayesian networks constitute a very attractive formalism for representing uncertain knowledge that has been successfully applied in different fields. However, Bayesian networks have been used in medicine essentially to assist in the diagnosis of disorders and to predict the natural course of disease after treatment (prognosis). A novelty of this work is the application of Bayesian networks to other, more management oriented, medical problems. The preliminary results obtained are encouraging, because the learned network is able to manage in a robust way, using a single model, a variety of different prediction problems.

For future works, we plan to extend and refine our model, including more variables, validate it taking into account expert knowledge and use it as a tool to assist to the hospital manager. We also plan to apply Bayesian networks to other management medical problems.

\section{References}

AC96. S. Acid, L. M. de Campos. An algorithm for finding minimum d-separating sets in belief networks. in: E. Horvitz, F. Jensen (Eds.), Proceedings of the Twelfth Conference on Uncertainty in Artificial Intelligence, Morgan Kaufmann, San Mateo, 3-10, 1996. 30 
AC01. S. Acid and L. M. de Campos. A hybrid methodology for learning belief networks: BENEDICT. Int. J. Approx. Reason., 27(3):235-262, 2001. 30

AC. S. Acid and L. M. de Campos. An algorithm for learning probabilistic belief networks using minimum d-separating sets. Submitted to J. Artif. Intell. Res. 30

CH92. G. F. Cooper and E. Herskovits. A Bayesian method for the induction of probabilistic networks from data. Mach. Learn., 9(4):309-348, 1992. 30

DH73. R. Duda, P. Hart. Pattern Classification and Scene Analysis. John Wiley and Sons, New York, 1973. 32

HGC95. D. Heckerman, D. Geiger, D. M. Chickering. Learning Bayesian networks: The combination of knowledge and statistical data. Mach. Learn., 20:197243, 1995. 30

Pea88. J. Pearl. Probabilistic Reasoning in Intelligent Systems: Networks of Plausible Inference. Morgan Kaufmann, San Mateo, 1988. 28

Qui93. J. R. Quinlan. C4.5 Programs for Machine Learning. Morgan Kaufmann, 1993. 32

SGS93. P. Spirtes, C. Glymour, R. Scheines. Causation, Prediction and Search. Lecture Notes in Statistics 81. Springer Verlag, New York, 1993. 30 